\title{
Turning limited experimental information into 3D models of RNA
}

\author{
SAMUEL COULBOURN FLORES and RUSS B. ALTMAN
}

Department of Bioengineering, Stanford University, Stanford, California 94305, USA

\begin{abstract}
Our understanding of RNA functions in the cell is evolving rapidly. As for proteins, the detailed three-dimensional (3D) structure of RNA is often key to understanding its function. Although crystallography and nuclear magnetic resonance (NMR) can determine the atomic coordinates of some RNA structures, many 3D structures present technical challenges that make these methods difficult to apply. The great flexibility of RNA, its charged backbone, dearth of specific surface features, and propensity for kinetic traps all conspire with its long folding time, to challenge in silico methods for physics-based folding. On the other hand, base-pairing interactions (either in runs to form helices or isolated tertiary contacts) and motifs are often available from relatively low-cost experiments or informatics analyses. We present RNABuilder, a novel code that uses internal coordinate mechanics to satisfy user-specified base pairing and steric forces under chemical constraints. The code recapitulates the topology and characteristic L-shape of tRNA and obtains an accurate noncrystallographic structure of the Tetrahymena ribozyme P4/P6 domain. The algorithm scales nearly linearly with molecule size, opening the door to the modeling of significantly larger structures.
\end{abstract}

Keywords: computational; constraint; folding; internal coordinates; structure prediction

\section{INTRODUCTION}

RNA plays a pervasive role in gene regulation and expression. Messenger RNA provides the template for protein synthesis but also forms structures to regulate that synthesis. The ribosome is composed primarily of RNA. The implications of RNA biology for human health are enormous. The regulatory elements in viral mRNA suggest attractive targets for antiviral therapies. Micro-RNAs represent a nascent theater of treatment. The ribosome has become critical as an antibiotic target with the emergence of drug-resistant bacteria. Research in these areas is continually hampered by the lack of a detailed understanding of the three-dimensional (3D) structure and dynamics of RNA.

Part of the challenge arises from the experimental difficulty of crystallizing a highly charged, very flexible molecule with a dearth of the distinctive surface features needed for recognition (Ferré-D'Amaré et al. 1998), and a propensity for kinetic traps. The difficulty of obtaining atomic coordinates contrasts with the greater ease with which base-

Reprint requests to: Samuel Coulbourn Flores, Department of Biology, Stanford University, Stanford, CA 94305, USA; e-mail: samuelfloresc@ gmail.com; fax: (650) 644-8416.

Article published online ahead of print. Article and publication date are at http://www.rnajournal.org/cgi/doi/10.1261/rna.2112110. pairing and stacking contacts and the general layout of helices and domains can often be obtained. For example, Levitt (1969) compiled tertiary base-pairing contacts of tRNA from mostly phylogenetic calculations. For the P4/P6 domain of the Tetrahymena group I intron, we can identify several motifs by sequence analysis (Costa and Michel 1995), while we know other tertiary contacts from structural bioinformatics (Lescoute and Westhof 2006), NMR, functional assays, and other experiments.

Several computational methods can predict the structures of RNAs that are sufficiently small. The nucleic acid builder (NAB) is a programming language that facilitates manipulation of RNA molecules, residues, and atoms; however, it has very limited ability to infer missing information such as the conformation of loop regions (Macke and Case 1998). Fragment assembly of RNA (FARNA) assembles structures by sampling trinucleotide fragments from a database and screens these using a coarse-grained potential that favors base pairing and stacking geometries (Das and Baker 2007). Similarly, MC-Sym samples four-residue cycles from a database that are consistent with known basepairing contacts and progressively builds up structure (Parisien and Major 2008). Both of these methods, however, have computer time requirements that scale poorly with size. The latter reproduced the structure of tRNA to 
3.1-3.8 ̊ root mean square deviation (RMSD), but only by applying base-pairing contacts known to exist from direct observation of the crystal structure (Gautheret et al. 1993; Major et al. 1993). Fragment assembly approaches are good at recovering local structure but not contacts across fragments, such as occur in protein $\beta$ sheets (Dong et al. 2008) or in RNAs with tertiary contacts (Parisien and Major 2008). Discrete molecular dynamics (DMD) has a simplified potential for RNAs that are represented as having only three pseudoatoms per residue; it reportedly solved the structure of tRNA (to 7.2 $\AA$ RMSD) but not larger molecules (Ding et al. 2008). Molecular dynamics (MD) computes the dynamics of atoms under a force field that approximately captures the important physical interactions; however, this method is too slow for the molecules treated here. The nucleic acid simulation tool (NAST) uses one pseudoatom per residue to represent an RNA structure and can fold the $(\sim 159$ residue $)$ P4/P6 domain to $16.3 \AA$ RMSD, but the characteristic parallel helices are not recapitulated (Jonikas et al. 2009). In prior work, we showed that RNABuilder can build the entire 200-residue Azoarcus ribozyme structure to $4.6 \AA$ RMSD using structural templates from the Twort and Tetrahymena ribozymes (Flores et al. 2010). However, other ribozymes, such as that from Bangia Fuscopurpurea, have entire domains with no close homologs of known structure, although the core and some peripheral elements can be fitted to existing templates. For such cases, there is a need for a modeling program that can use experimentally observed contacts to build a model of an entire RNA domain in the absence of observed atomic coordinates. In addition, the method should scale up to larger systems in a reasonable way.

RNABuilder uses the Simbody internal coordinate mechanics (ICM) library, which computes dynamics in order$\mathrm{N}$ time (proportional to the number of bodies). The fastestoscillating bond lengths in the system limit the maximum size of the integration time step in a Cartesian-coordinate MD simulation, but under ICM these bond lengths can be fixed allowing a longer time step. The bond angles are typically also fixed, leaving dihedral torsions as the only degrees of freedom. It is also possible to freeze bond torsion angles, effectively turning the two atoms connected by the bond into a single body, reducing the number of bodies and thus the computational expense. Bonds can be rigidified for arbitrary regions or even entire molecules; this also means fewer force evaluations since there is no need to calculate forces between two points of the same rigid body. The key to RNABuilder's versatility is its ability to enforce base pairs of any of the numerous types cataloged by Leontis et al. (2002), as well as stacking and other interactions. This feature is again possible because of ICM. ICM supports bodies that have all six rigid-body degrees of freedom, rather than the three translational ones associated with point atoms in most MD codes. This allows us to fully specify the position and orientation of one base with respect to another in a base-base interaction of a given type. The RNABuilder force field applies forces and torques to enforce these relative positions and orientations (Flores et al. 2010).

RNABuilder is in some ways a descendant of the Gō model (Taketomi et al. 1975). In its original instantiation, the Go model consisted of a two-dimensional coarse-grained protein whose residues only interacted with residues that would be nearest neighbors in the native state. Residues were limited to discrete sites on a rectangular lattice. Sterics prevented more than one residue from occupying a single site, and chemistry fixed the distance between consecutive residues. This model was used to study the folding thermodynamics of proteins (Taketomi et al. 1975). In RNABuilder, the user is free to apply knowledge of the base-pairing contacts (which may be obtained by various theoretical or experimental means) rather than explicit knowledge of the full 3D structure of the native state. Sterics are treated approximately by collision-detecting spheres as we will explain, while ICM guarantees chemically reasonable bond lengths, angles, and connectivities.

RNABuilder differs most notably from fragment assembly methods in that it is a dynamical code, which uses forces to bring bases into desired positions, rather than assembling rigid fragments. Fragment assembly methods do well at reproducing local structure, but closing long-range tertiary contacts requires that multiple fragments line up in just the right way; the probability of this occurring with a finite-size fragment library is small.

RNABuilder differs from other dynamical codes such as MD or DMD in that all forces are user specified, making our code a powerful platform for combining experimental constraints and user hypotheses. It shares with MD a close support of popular optimization techniques, such as simulated annealing, under which an initially high temperature is gradually decreased (Kirkpatrick et al. 1983). The time required for convergence, including time spent escaping kinetic traps, can be related to the experimental folding time (Snow et al. 2002), which in turn depends on secondary and tertiary structural contacts (Nkwanta and Ndifon 2009).

In this work we model the structure of a 76-residue tRNA molecule, following a tradition of using this molecule as a benchmark (Major et al. 1993). We use contacts obtained only from experiments that preceded or do not make explicit reference to the crystallographic coordinates. The resulting structure has a resolution comparable to that obtained with other methods. We repeat this process using base-pairing contacts obtained from examination of the known 3D structure and obtain a further improvement. We then use published NMR, phylogenetic, structural bioinformatic, functional assay, and cross-linking data to apply basewise forces to build a model of the 160-residue Tetrahymena ribozyme P4/P6 domain. These data are "extra-crystallographic" in the sense that they were obtained without explicit reference to the crystallographic coordinates. Applying all these forces results in a structure that aligns with the 
crystallographically observed one to an accuracy that exceeds that of previously announced methods. To show how the structure would improve given perfect information, we repeat the process using base-pairing contacts obtained by examination of the crystallographically observed 3D structures, generating what we call a "crystallographic model."

\section{RESULTS}

RNABuilder flags base interactions as successfully enforced if the glycosidic nitrogen of one base is within a useradjustable distance $(1 \AA$ here $)$ of its ideal position with respect to the base it is paired with. For the extracrystallographic model of tRNA, we applied 50 interactions including secondary structural Watson-Crick base pairs, tertiary base pairs, and stacking interactions, as summarized in Supplemental Table S1. RNABuilder automatically added 35 offset-stacking interactions between consecutive bases in the duplexes. It successfully enforced 77 of the 85 total base interactions, including 43 of the 50 manually applied ones as shown in Supplemental Table S1. Of the eight total contacts that failed to form, seven were part of a redundant-forces approach to enforce contacts (base-pairs 26-44, 15-48, 19-56, and 8-14) of ambiguous type and were partially successful in their objective as we will explain in the Discussion. Only one (a helical stacking contact between residues 7 and 8) was an outright failure. During the last, converged, stage the RMSD varied from 8.1 to $11.1 \AA$, with an average of $9.6 \AA$, with respect to the experimentally observed (Sussman et al. 1978) coordinates (Fig. 1). We also used the interaction network fidelity method's Matthews correlation coefficient (MCC) (Parisien et al. 2009) as an additional metric of comparison. The MCC in this case calculates a correlation between the predicted and crystallographically observed base-pairing interactions. The MCC of the last structure in this trajectory was $74 \%$.

The crystallographic model does not have the redundant contacts but does have additional contacts obtainable only by examining the experimentally obtained coordinates. This model has 87 total base contacts, of which 85 formed correctly. Only one of the manually applied contacts failed, as we will discuss later. During the last stage the RMSD varied from 4.4 to $8.4 \AA$, with an average of $6.1 \AA$. The MCC of the last structure was $80 \%$.

The extra-crystallographic model of P4/P6 had 166 base contacts, all of which were enforced successfully, most of them in the first $2 \mathrm{nsec}$ of an 8-nsec simulation (Supplemental Table S2). In the latter $4 \mathrm{nsec}$ of the simulation, the structure had RMSD with respect to the experimentally observed structure ranging from 8.7 to $11.3 \AA$, with an average of $10.0 \AA$, making it the most accurate computational recapitulation of this molecule made to date without explicit reference to crystallographic results (Fig. 2). The MCC of the last structure was $66 \%$.

The crystallographic model of P4/P6 had 185 base contacts, of which 181 were enforced successfully. In the final stage the structure had a 9.4-10.0 $\AA$ RMSD, with an average of $9.6 \AA$. The MCC of the last structure was $71 \%$.

To determine whether the accuracy was limited by sampling or by the force field, we started from the crystallographically observed structure and allowed the model to relax under the crystallographic contacts until equilibration. We obtained a structure with $4.2 \AA$ RMSD with respect to the crystallographically observed one. This suggests that some of the deviation from the crystal is due to incomplete sampling, but more is due to bias in the force field. Similarly the observed structure under extra-crystallographic contacts relaxed to $7.8 \AA$.

For P4/P6 we again let the crystallographically observed structure relax under the crystallographic contacts, and obtained an $8.4 \AA$ structure. Under the extra-crystallographic contacts it relaxed to a $10.4 \AA$ RMSD (Fig. 3).

In order to show the dependence of our model on the quality of our experimental constraints, we resolved the extra-crystallographic model with different historical conditions, with the aim of showing how the structure improved

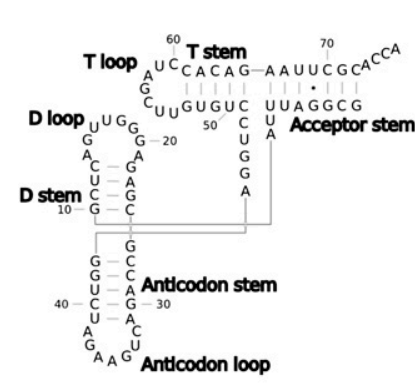

A. Secondary structure

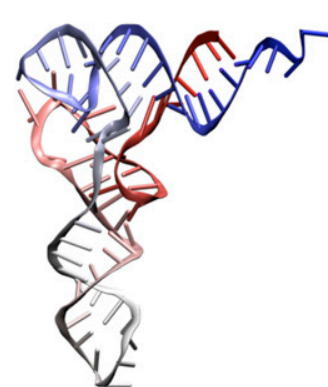

B. Experimentally Observed
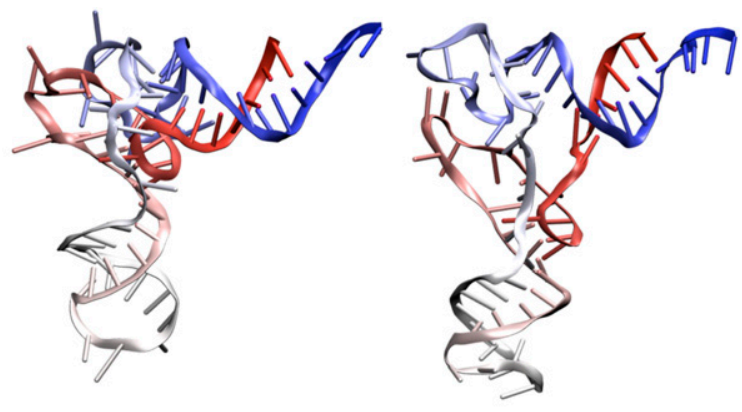

C. Extra-crystallographic model D. Crystallographic
model

FIGURE 1. tRNA. The observed structure $(B)$, models generated using extra-crystallographic $(C)$, and crystallographic $(D)$ contacts. Domains in the secondary structure diagram $(A)$ are oriented roughly as they are in the $3 \mathrm{D}$ structures $(B-D)$. $B-D$ are colored by residue number, with red being the lowest and blue being the highest. Note the improved structural similarity between $D$ and $B$, as compared to that between $C$ and $B$. 


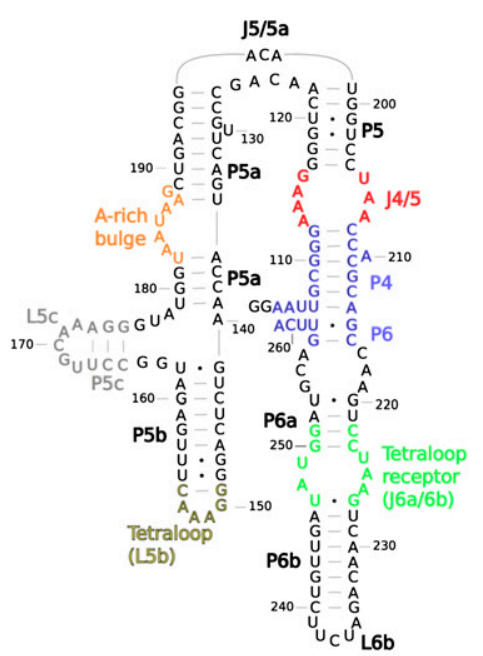

A. Secondary structure

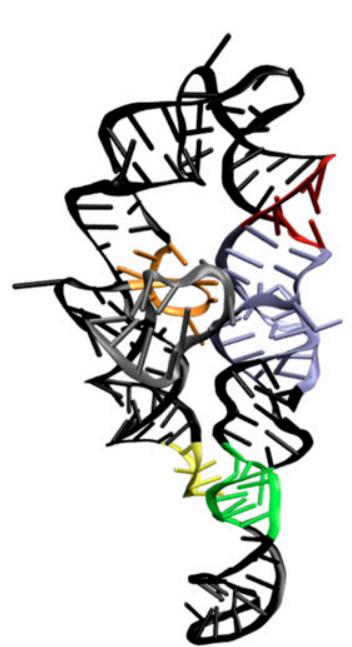

B. Experimentally Observed

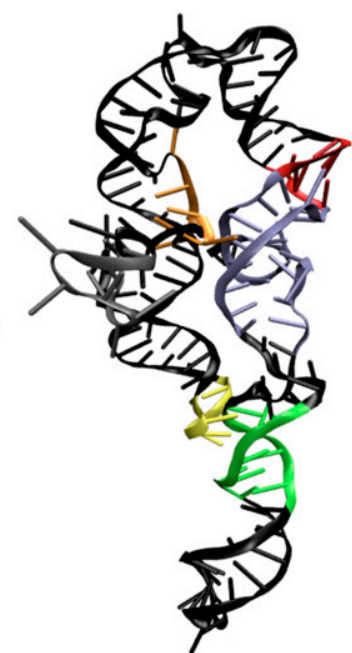

C. Extra-crystallographic model

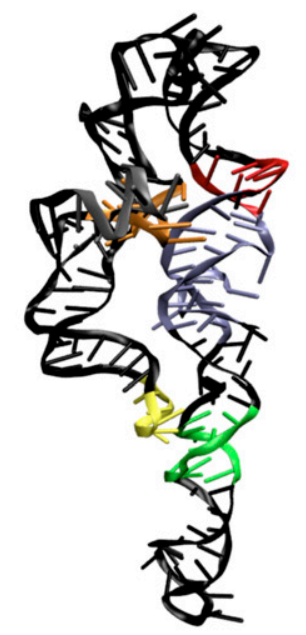

D. Crystallographic model

FIGURE 2. P4/P6. Models generated using extra-crystallographic $(C)$ and crystallographic $(D)$ contacts, compared to the experimentally observed (B) structure. Regions of particular interest are colored consistently across $A-D$, following the color scheme of Cate et al. (1996a).

as key discoveries were made. We solved structures with data that were available in 1988, 1994, 1997, 2005, and 2006. As before, we ignored all data that made explicit use of the crystal structures of P4P6 (Cate et al. 1996a). Remarkably, the 1994 structure had a $13.4 \AA$ RMSD (Fig. 4) and a finalstructure MCC of $61 \%$. We explain the historical and scientific significance of these results in the Discussion. Similarly, we ran a different simulation with randomly removed contacts to show robustness and found that removing $10 \%$ and $30 \%$ of the contacts increased RMSD by 1.5 and $10.0 \AA$, respectively, compared with using $0 \%$ missing contacts. Thus in addition to the tolerance for ambiguity in world knowledge, the method has a fair tolerance for contacts, which might be missed due to user error.

\section{DISCUSSION}

\section{tRNA}

The tRNA structure largely folded successfully, recapitulating the salient features of the molecule. Most applied base pairs were successfully enforced. The overall layout of the helices is correct, as is the topology. In the extra-crystallographic model, all but one of the failed base interactions were part of our strategy to deal with ambiguous contacts (see Materials and Methods). Of the failed interactions in the crystallographic model, two were part of the structurally important network connecting T- and D-loops; we discuss these in detail.

\section{Ambiguous contacts in the extra-crystallographic structure}

As mentioned, the 44-26 base-pair (bp) type was unknown so we applied Watson-Crick/Hoogsteen and Watson-
Crick/Watson-Crick cis contacts (the trans is geometrically infeasible); the correct contact (the Watson-Crick/ Watson-Crick one) was recapitulated. This strategy failed to recapitulate the right interaction type for $19-56$ or 8-14, but the bases were at least brought into contact in both cases. The 15-48 bp has the right edges interacting, although the relative angular orientation of the two bases is inaccurate. Thus, in one case we were completely successful in recovering the correct type of contact when this was not given, and in three cases we generated a contact although it did not recapitulate the correct type. The partial success of

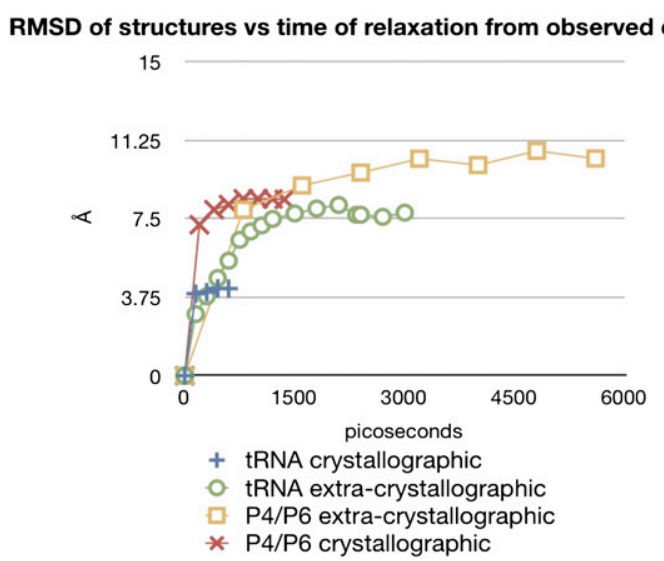

FIGURE 3. Relaxation of structures. The atomic coordinates of tRNA were set to those experimentally observed and the dynamics were computed at $10 \mathrm{~K}$ under the crystallographic (blue plus signs) and extra-crystallographic (green circles) forces. The same was done with P4/P6 (orange squares and red crosses). The structures drifted away from the initial configuration and equilibrated at a structure with RMSD somewhat lower than that of the respective predicted structures. 


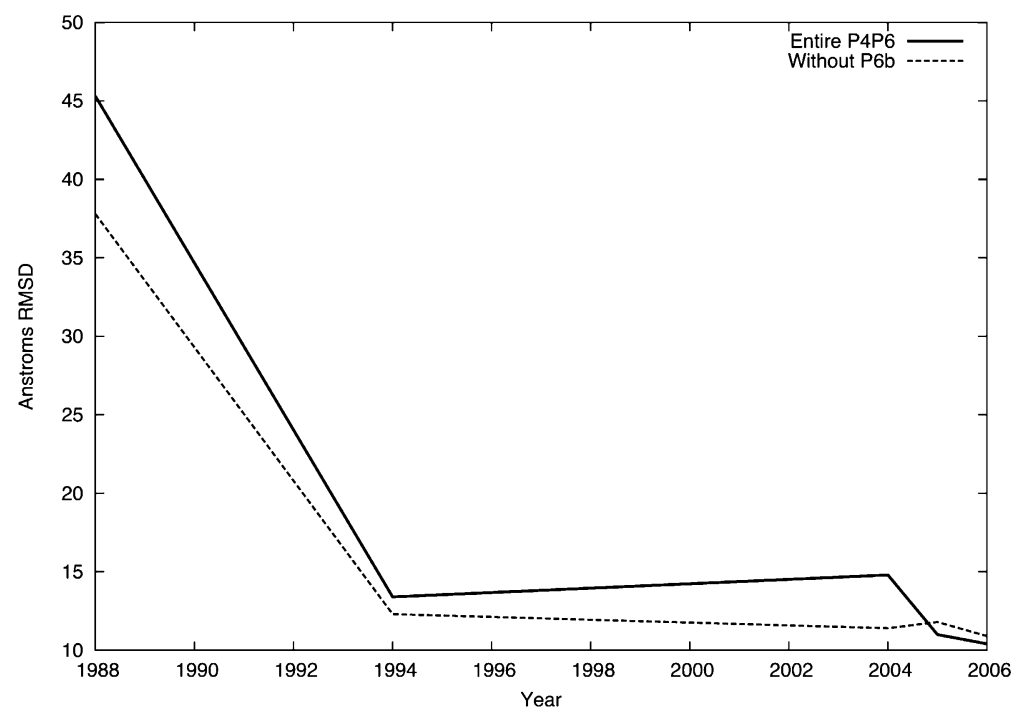

FIGURE 4. P4P6 structures from historical contacts. We created models using data that were available to investigators at crucial historical junctures, starting with the publication of the secondary structure in 1988 and ending with a statistical study of three-way junctions in 2006 (see Discussion). The graph shows the RMSD that would have been obtained with respect to the crystal structure using the historical constraints. In 1988 no tertiary contacts were known and so RMSD is high. Without knowing about the A-platform in J6a/6b, P6b was mobile, and so before the 2005 constraints the structures aligned without P6b (dashed line) had lower and less variable RMSD than those with P6b (solid line).

enforcing these contacts in the extra-crystallographic model became a full success once the ambiguity was removed in the crystallographic model.

\section{Base-stacking sandwich in crystallographic structure}

As mentioned, a highly conserved base-stacking sandwich was applied to enforce the contact between the T- and D-loops. Bases 57-18 and 57-19 interaction, but nonetheless the respective loops came together under the action of the remaining, successful contacts. The base-stacking sandwich is the primary reason the crystallographic structure is more accurate than the extra-crystallographic; we did not notice much improvement from adding the other mentioned crystallographic contacts.

\section{P4/P6}

In the P4/P6 extra-crystallographic structure, remarkably, we correctly enforced all 166 applied base interactions. Further, we accurately recapitulated three contacts that can be observed in the experimental coordinate set but were not part of our model, and partially recapitulated six more. In the crystallographic model we successfully enforced 181 out of 185 applied contacts; the unenforced contacts were not crucial to forming a large-scale structure. Much as before, we accurately recapitulated two stacking interactions that were neglected in the model and partially recapitulated four more (Supplemental Table S2).
We also asked the question, how well would our model work with less information? We placed ourselves in the context of crucial junctures in Tetrahymena ribozyme research. In 1988 the secondary structure was published. No tertiary contacts were known, so the RMSD is high at $45 \AA$ (Fig. 4). By 1994, the crucial contact between L5c and J6a/6b was known, although it was off by a few bases. Remarkably, RNABuilder solved the structure to a $13.4 \AA$ RMSD using these constraints. In 1996 the structure was solved crystallographicallybut we ignore this. In 1997, constraints on single-residue bulges were described (Zacharias and Sklenar 1997). In 2004, the structure of $\mathrm{J} 4 / 5$ was solved by nuclear magnetic resonance (NMR) (Znosko et al. 2004), but our continued presumed ignorance of the A-platform motif, discovered crystallographically in P4P6 (Cate et al. 1996b), continued to let P6b take random orientations, leading to a slightly higher RMSD of $14.8 \AA$. In 2005, the tetraloop-receptor motif was solved by NMR (Davis et al. 2005)—-these constraints lowered RMSD to $11.0 \AA$. In 2006, the topology of threeway junctions was described statistically (Lescoute and Westhof 2006), at which point our model dropped to a $10.4 \AA$ RMSD, and an MCC of $64 \%$. We point out that it is probably impossible for the RMSD to drop to zero, because $\mathrm{L} 5 \mathrm{c}$ in the reference structure is contacting a crystal neighbor, making the position of P5c incorrect (Cate et al. 1996a).

A significant amount of information is available about the structure of the Tetrahymena ribozyme that was or could have been known in the absence of crystallographic coordinates. We translated this information into forces that drive the model to recover the structural features of the P4/P6 domain. In this way we demonstrate RNABuilder's usefulness as a platform for combining multiple experimental constraints to determine 3D structure.

\section{Sampling or force field?}

In our relaxation experiments, we found that if the initial atomic coordinates were taken from the experimentally observed structure, the model would drift away from those coordinates, although in all cases it equilibrated to a structure that was of slightly better quality (in terms of RMSD) than the structure predicted de novo. If the force field were perfect, and all accuracy problems were due to incomplete sampling, then the structure would not have drifted from the initially correct configuration. Because it did, we can 
surmise that the force field offers significant room for improvement. The geometric parameters of the base-pairing forces were taken from observed base pairs (Leontis et al. 2002). However, a single observation does not represent the range and nature of the fluctuations that can occur in a base pair. Thus the method would benefit from improved parameters (which are user adjustable) and/or a reformulation of the interaction force. In addition the sterics are currently handled by nonoverlapping spheres on a limited number of atoms; a higher-order approximation to the correct steric interactions between atoms would improve accuracy but also involve higher cost. Finally, the flexibility of the bonds, which in most cases have fixed bond lengths and angles, could be increased, albeit again at significant additional cost.

\section{CONCLUSIONS}

As mentioned, RNABuilder can thread an RNA of unknown structure onto multiple templates from structurally related RNAs to obtain an accurate prediction (Flores et al. 2010). In this work we showed that the program is also useful for modeling structures when no template is available. We can use base-pairing contacts and other information that was, or could have been, known in the absence of structural coordinates to generate structures of tRNA and P4/P6. We recapitulated the topology and characteristic L-shape of tRNA, and the best published noncrystallographic P4/P6 structure in this way. To demonstrate how additional information can provide further improvements in fidelity, we then added contacts obtained directly from the crystal structure, and obtained dramatically improved structures for tRNA and P4/P6. With this work we prepare the ground for modeling larger structures for which partial experimental information is available.

\section{AVAILABILITY}

Binary distributions (for Linux, Mac, and Windows) of RNABuilder are available for download from the RNAToolbox project on the Stanford Simbios Center's website, SimTK.org (http://simtk.org/home/rnatoolbox). We also provide a source code and a tutorial. Simbios provides software support and workshops. Calculations in this work used RNABuilder revision 277, Molmodel revision 680, and Simbody revision 1027, except for the historical calculation that was done with RNABuilder revision 284. The input file for the 2006 historical P4P6 run is provided as a supplemental file.

\section{MATERIALS AND METHODS}

\section{Extra-crystallographic contacts in tRNA}

The structure of tRNA was solved so early (Kim et al. 1973; Robertus et al. 1974) that it predates many methods of determining base- pairing contacts. Nonetheless, the pre-1974 literature is full of correct predictions of base-pairing and other contacts. Most tertiary contacts in RNA are predicted by coevolution, a technique manually employed in early work (Levitt 1969). The field has progressed significantly since that time (Gautheret et al. 1995), and yet has added surprisingly few tRNA tertiary contacts despite the $(\sim 100$ fold) explosion in available sequences. In this work we divide the structural information into two classes: extra-crystallographic and crystallographic. The extra-crystallographic contacts are those that were known before the 3D structure of tRNA was obtained in 1974, plus those that were discovered after 1974 without explicitly referring to the crystallographic coordinates. We obtained the crystallographic contacts by visual examination of the crystallographically observed 3D structure ("structure gazing") and by using RNAView (Yang et al. 2003), a program that searches for occurrences of base pairs of known types (Leontis et al. 2002) in the 3D crystal structure.

\section{Secondary structure}

The cloverleaf secondary structure of tRNA was one of three proposed when the first (yeast alanine) tRNA sequence was determined (Holley et al. 1965), and was confirmed by sequence comparison when the yeast tyrosine (Madison et al. 1966) and phenylalanine (RajBhandary et al. 1966) tRNAs were sequenced. Levitt aligned 14 sequences to determine the secondary structure for his 1969 model (Levitt 1969); remarkably this was nearly identical to that published by Gutell 23-yr later based on 1710 aligned sequences (Gutell et al. 1992). Notably Gutell et al. (1992) detected the noncanonical G-A base pair between residues 26 and 44, which Levitt (1969) did not. We used the Gutell et al. (1992) predictions in this work.

\section{Tertiary base pairs}

Isolated contacts are harder to detect than helices. First, they do not occur in runs as do helical segments and so cannot be detected by simple sequence complementarity. Second, they do not coevolve as strictly as helical base pairs because tertiary contacts can occur in a variety of ways. Nonetheless, the crucial (Major et al. 1993) 8-14 bp were known and verified by a photoreaction experiment (Levitt 1969). Base pairs 15-48 and 19-56 were known by Levitt (1969) and later by Gutell et al. (1992). Accordingly we applied all of these contacts. Levitt also reported a number of base pairs that were implied by his theoretical model rather than by experiments; we did not use these in our work.

In many cases even if two bases are known to be in contact, the type of interaction within the Leontis-Westhof classification is not known. We deal with this by applying all feasible interactions between the two residues. Similarly in some cases the user may not know which residues are in contact; this ambiguity can potentially be dealt with by applying base-pairing forces between multiple pairs of residues hypothesized to be in contact, although we do not attempt that in this work. In either case the bases will then interact in a potential that has multiple minima corresponding to the applied interactions.

\section{Base triples}

Base triples, like isolated base pairs, are more difficult to detect than helices. For triples, the tertiary contact has additionally been observed to shift in position, thus diluting simple measures 
of covariation. Accordingly, Gautheret et al. (1995) proposed that triplets should be detected by computing a covariation score not only between the putative contacting residues, but also between neighboring pairs (Gautheret et al. 1995). Their test predicts the base triples (13•22) 46 and $(12 \cdot 23) \bullet 9$ with high confidence, and $(11 \cdot 24) \cdot 36$ and $(10 \cdot 25) \bullet 45$ with lower confidence. Of these, only $(11 \cdot 24) \cdot 36$ is incorrect. The triple $(12 \cdot 23) \bullet 9$ was known to Levitt (1969). The existence of this triple is corroborated by later mutational experiments showing that mutating 24 to make the $(11 \cdot 24) \cdot 9$ interaction preferable, or mutating 9 to make neither triple possible, leads to the same misread phenotype (Smith and Yarus 1989). Residue 36 is part of the anticodon and is unavailable for a structural triple (Fuller and Hodgson 1967), and the apparent coevolution of $(11 \cdot 24) \cdot 36$ can be explained by the mentioned mutagenesis experiments (Smith and Yarus 1989). The (10•25)•45 triple is difficult to detect because 45 is almost invariant (Fuller and Hodgson 1967); in particular it was not detected by Levitt (1969), or by Klingler and Brutlag (1993) (Altman 1993). We therefore believe the triples $(13 \cdot 22) \bullet 46$ and $(12 \cdot 23) \bullet 9$ would be known today even without crystallographic coordinates, but $(11 \cdot 24) \cdot 36$ would have been discarded and $(10 \cdot 25) \bullet 45$ would have required corroboration with experiments that have not been performed to our knowledge. With this in mind we consider only the (13•22)•46 and $(12 \cdot 23) \cdot 9$ triples as extra-crystallographic.

\section{Helix stacking}

Helices tend to stack end to end to maximize $\pi$-orbital interactions. For tRNA, Levitt (1969) correctly predicted that the D should stack on the anticodon, and the T should stack on the acceptor arm. This is the only arrangement in which each stack shares one uninterrupted strand, and only one of the two has a single residue that is stacked but not base paired.

\section{Crystallographic contacts in tRNA}

The crystallographic contacts we used to construct the tRNA model with RNABuilder include (1) those already detected and fully characterized extra-crystallographically; (2) those detected and correctly characterized by RNAView; (3) those observed only by structure gazing; and (4) those observed extra-crystallographically or by RNAView, but were ambiguous or incorrect in their details and were subsequently corrected by structure gazing.

Some contacts were detected automatically by RNAView, including base pairs 9-23, 18-55, 19-56, 48-15, and 54-58. A few additional contacts come from structure gazing. In particular, there is a base-stacking "sandwich" connecting the D and T loops, which is structurally conserved (F Major, pers. comm.), linking residues 58-18-57-19. In three cases (9-23, 10-45, and 44-26) a contact between a pair of residues was known, but the specifics of the interaction could not be known without referring to the structure. One contact, 15-48, was detected by RNAView but assigned the wrong type. It was also known extra-crystallographically but the type was ambiguous. Finally, there is a stacking interaction between 48 and 59, which RNAView does not detect.

\section{Extra-crystallographic contacts and motifs in P4/P6}

In modeling P4/P6, we sought to demonstrate RNABuilder's ability to incorporate multiple contacts to accurately reconstruct an entire 160-residue domain. All contacts used are pre- or extracrystallographic, to demonstrate that RNABuilder can build structure in the absence of full structural coordinate data. Below, we detail the information provided to RNABuilder to construct the model, beginning with the distal end of P6 and proceeding up the helix, around the hinge at J5/5a, and down to P5b.

\section{Secondary structure}

The secondary structure of the group I intron is known mostly from coevolution calculations, which were first done by Michel et al. (1982) and were refined almost exactly into the form known today by Cech (1988). For the P4/P6 domain, the latter work is missing only the closing base-pair 141-162, an omission that was corrected by Murphy et al. (1994).

\section{Eleven-nucleotide motifs in P6}

The internal loops connecting P6b to P6a and P6a to P6 are 11-nucleotide (nt), or A-platform, motifs as described by Doudna and collaborators (Cate et al. 1996a,b; Batey et al. 1999). The 11-nt motif was discovered by sequence analysis of group I and group II introns (Costa and Michel 1995). It was later observed structurally in the Tetrahymena ribozyme (Cate et al. 1996a,b; Batey et al. 1999), as well as other related and unrelated molecules. Last, it has been engineered and studied in isolation (Davis et al. 2005). We examined the Davis construct to obtain the base-pairing contacts needed to fully specify an 11-nt motif. We then applied these contacts to construct the two 11-nt motifs in our model.

\section{Helical stacking of P4 and P6}

Murphy et al. (1994) fused helices P4 and P6, found that all tertiary contacts were maintained and folding was sped up, and concluded that these helices are stacked. Accordingly, we applied a helical stacking interaction, described below, between residues 214 and 215 to ensure P4 was coaxial with P6.

\section{Single-residue bulges}

Single residue bulges can be either extruded or inserted into the helical stack. In either case the interhelical angle they induce is small, ranging from $10^{\circ}$ to $20^{\circ}$ (Zacharias and Sklenar 1997) There is a single-residue bulge at residue A210; we treated it by enforcing a helical stacking interaction between residues 110 and 111 on the opposing strand, which should result in a small interhelical angle. There are similar bulges at residues 130 and 188, which were treated in precisely the same way. RNABuilder automatically recognizes three or more consecutive base pairs as helical and applies the corresponding offset-stacking force. However residue 210 interrupts helix P4 leaving two isolated base pairs, and so we explicitly applied a helical stacking interaction between residues 111-112 and 208-209.

\section{Structure of $\mathrm{J4/5}$}

NMR experiments on the J4/5 internal loop (Znosko et al. 2004) showed that residues 114 and 206 are in a sheared interaction, as are 207 and 113. Residues 205 and 116 form a noncanonical Watson-Crick base pair. This means base 115 must be either 
inserted or extruded, and in either case the P4/P5 interhelical angle must be small (Zacharias and Sklenar 1997). We modeled 115 as inserted by stacking it with 114 and 116 .

\section{Hinge at $J 5 / 5 a$}

Significant evidence exists that the J5/5a loop is specifically designed to promote folding and is more than a passive hinge. In particular, Szewczak and Cech (1997) tested engineered P4/P6 molecules with variations in the J5/5a loop and found that all mutants required more $\mathrm{Mg}^{2+}$ than the wild type to induce folding, but the more disorder-prone sequences required less than the more order-prone ones; in particular a double-helical J5/5a variant used as a control did not fold at all. The group I intron from Pneumocystis carinii has what appears to be a variant of the 11-nt motif in J5/5a, and it promotes folding; however, the influence is less than that of long-range tertiary interactions (Ikawa et al. 2000). In conclusion, the feature of J5/5a most important for folding P4/P6 is that it acts as a hinge. In any event we found no indication in the literature that specific base-pairing contacts within J5/5a were known before the structure was solved. For these reasons we applied no forces to the residues in J5/5a.

\section{A-rich bulge}

Prior to solution of the P4/P6 structure it was not known whether the A-rich bulge interrupted the P5a helix. Luebke and Tinoco (1996) measured an interhelical angle of $69^{\circ} \pm 5^{\circ}$ for the native sequence using gel electrophoresis. However, residual dipolar coupling experiments in the Al-Hashimi laboratoy have shown that the interhelical angle about the three-residue bulge in TAR depends strongly on the type and concentration of ligands and counterions (Casiano-Negroni et al. 2007), in particular, at high salt the angle is small. There are more general arguments in favor of helix stacking in P5a. First, helix stacking is energetically favored between helices separated by short loops-in this case opposite the bulge is a loop of zero length (Lescoute and Westhof 2006). P5a stacking would permit the P5a and P5c helices to pack against the P5-P4-P6 stack, which should be favored by saltmediated interactions. The same packing would result in the base normals of all helices except P5c pointing in parallel directions, a preferred configuration according to Laederach et al. (2007). Given the debate on this issue we did not explicitly enforce an interhelical angle here.

There is one inferred tertiary contact in the A-rich bulge. A dimethyl sulfate treatment on the double mutant C109G-G212C in $\mathrm{P} 4$ resulted in a modified methylation pattern in the adjacent base pairs, and at residue A183 in the A-rich bulge. The single mutant G212C eliminated splicing activity almost completely. Based on this and other experiments Flor et al. (1989) concluded that A183 is involved in a base triple via the minor groove of C109-G212, but did not further specify what type of interaction was involved. Because this fits the pattern of the type I A-minor interaction (Nissen et al. 2001) perfectly, we enforced this contact with a trans sugar-edge base pair between residues 212 and 183. In fact, this contact is not correct-residue 183 became unprotected when residue 212 was mutated but only because residue 184 was protecting the former when paired with 212 . The crystal structure revealed a type I A-minor interaction at base-pair 109-212, but it is with residue 184 rather than 183 (Cate et al. 1996a).

\section{The P5abc three-way junction}

The last helix stacking decision is in the P5abc junction. To our knowledge, prior to Cate's structure no direct experimental information indicated the existence of helical stacking or tertiary contacts in this junction. Perhaps fittingly it has since been used as a test case for theoretical methods of predicting the geometry and topology of three-way junctions (Lescoute and Westhof 2006; Tyagi and Mathews 2007). P5abc belongs to family A according to the Lescoute and Westhof (2006)classification. According to this, P5a should stack on P5b, the longest loop should interact with P5a, and P5c should be roughly perpendicular to the stack. The stacking (residues 139-140) is correct, but the tertiary contact (between 174 and 178) is not; we nonetheless enforced both interactions. We did not enforce the vague guideline for the P5c orientation. P5c is known to contact P2 (Ikawa et al. 2000; Russell and Herschlag 2001), but of course P2 is outside the P4/P6 domain so this was not a usable constraint.

\section{L5C-P6 tetraloop-receptor interaction}

This crucial interaction closes P4/P6 into its final form. The contact was reported by Murphy et al. (1994), but was incorrectly predicted to be between residues 153 and 223. GNRA tetraloops and 11-nt receptor motifs were discovered by sequence analysis in group I introns (Costa and Michel 1995), and have since been observed in other molecules (Martick and Scott 2006). GNRA tetraloops have a characteristic structure with shared elements (Correll and Swinger 2003) and in particular the structure of the GAAA tetraloop has been solved in complex with the 11-nt receptor by Davis et al. (2005), providing us with a template to model both motifs accurately.

L5c is a GAAA tetraloop, and as discussed earlier there are two 11-nt receptors on P6. Murphy and Cech (1994) discovered that a single base substitution in L5c disrupted Fe(II)-EDTA protection in P6a, and correspondingly a single base substitution in P6a disrupted protection in L5c; from these experiments they concluded that residue A153 forms a base triple with C223-G250. and is corroborated by the Davis construct (Davis et al. 2005). We modeled this by applying a sugar-edge/sugar-edge/trans interaction between A153 and G250 consistent with a type I A-minor interaction (Nissen et al. 2001). We also applied a Watson-Crick/ Watson-Crick/trans interaction between 151 and 248 following Davis et al. (2005).

As we have explained, many of the contacts gleaned from post1996 publications could have been obtained even if the structure of the domain had never been solved. In all cases suitable RNABuilder interactions exist to enforce the contacts.

\section{Crystallographic contacts in P4/P6}

The crystallographic contacts, as before, come from RNAView or structure gazing, and in some cases consist of correcting an incorrect extra- or precrystallographic contact.

RNAView annotation gave us many contacts, namely, 183-212, 260-212, 188-168, 135-187, 184-212, 181-186, 139-164, 140163, 124-201, 123-198, 260-108, 10-259, 258-105, 257-104, and 156-103. RNAView does not detect stacking interactions, but we observed one between 183 and 184. The contact from the LescouteWesthof junction Family A (residues 183-212), used in the extracrystallographic run, is actually between 184 and 212 and was 
corrected in the crystallographic run. There are also three helical or near-helical contacts that were unknown before the structure was solved, namely, 139-164, 140-163, and 162-141(Cate et al. 1996a).

\section{SUPPLEMENTAL MATERIAL}

Supplemental material can be found at http://www.rnajournal.org.

\section{ACKNOWLEDGMENTS}

This work was supported by the Simbios National Center for Biomedical Computation, an NIH roadmap grant NIH GM072970. Joy $\mathrm{Ku}$ provided critical reading of this work. Charles Janac helped with testing RNABuilder on small molecules. Michael Levitt provided useful insights and discussion. Tom Cech explained the 212-184 contact in the context of protection experiments, as well as crystal packing artifacts in P4P6. We thank Chris Bruns, Peter Eastman, Michael Sherman, and Jeanette Schmidt for supporting and modifying the Simbody code as needed to make our software.

Received February 2, 2010; accepted May 24, 2010.

\section{REFERENCES}

Altman RB. 1993. Probabilistic structure calculations: A three-dimensional tRNA structure from sequence correlation data. Proc Int Conf Intell Syst Mol Biol 1: 12-20.

Batey RT, Rambo RP, Doudna JA. 1999. Tertiary motifs in RNA structure and folding. Angew Chem Int Ed Engl 38: 2326-2343.

Casiano-Negroni A, Sun X, Al-Hashimi HM. 2007. Probing $\mathrm{Na}^{+}-$ induced changes in the HIV-1 TAR conformational dynamics using NMR residual dipolar couplings: New insights into the role of counterions and electrostatic interactions in adaptive recognition. Biochemistry 46: 6525-6535.

Cate JH, Gooding AR, Podell E, Zhou K, Golden BL, Kundrot CE, Cech TR, Doudna JA. 1996a. Crystal structure of a group I ribozyme domain: Principles of RNA packing. Science 273: $1678-1685$.

Cate JH, Gooding AR, Podell E, Zhou K, Golden BL, Szewczak AA, Kundrot CE, Cech TR, Doudna JA. 1996b. RNA tertiary structure mediation by adenosine platforms. Science 273: 1696-1699.

Cech TR. 1988. Conserved sequences and structures of group I introns: Building an active site for RNA catalysis-a review. Gene 73: 259-271.

Correll CC, Swinger K. 2003. Common and distinctive features of GNRA tetraloops based on a GUAA tetraloop structure at $1.4 \AA$ resolution. RNA 9: 355-363.

Costa M, Michel F. 1995. Frequent use of the same tertiary motif by self-folding RNAs. EMBO J 14: 1276-1285.

Das R, Baker D. 2007. Automated de novo prediction of native-like RNA tertiary structures. Proc Natl Acad Sci 104: 14664-14669.

Davis JH, Tonelli M, Scott LG, Jaeger L, Williamson JR, Butcher SE. 2005. RNA helical packing in solution: NMR structure of a $30 \mathrm{kDa}$ GAAA tetraloop-receptor complex. J Mol Biol 351: 371-382.

Ding F, Sharma S, Chalasani P, Demidov VV, Broude NE, Dokholyan NV. 2008. Ab initio RNA folding by discrete molecular dynamics: From structure prediction to folding mechanisms. RNA 14: 1164-1173.

Dong Q, Wang X, Lin L. 2008. Prediction of protein local structures and folding fragments based on building-block library. Proteins 72: 353-366.

Ferré-D’Amaré AR, Zhou K, Doudna JA. 1998. Crystal structure of a hepatitis delta virus ribozyme. Nature 395: 567-574.

Flor PJ, Flanegan JB, Cech TR. 1989. A conserved base pair within helix $\mathrm{P} 4$ of the Tetrahymena ribozyme helps to form the tertiary structure required for self-splicing. EMBO J 8: 3391-3399.
Flores S, Wan Y, Russell R, Altman RB. 2010. Predicting RNA structure by multiple template homology modeling. Pac Symp Biocomput 2010: 216-227.

Fuller W, Hodgson A. 1967. Conformation of the anticodon loop intRNA. Nature 215: 817-821.

Gautheret D, Major F, Cedergren R. 1993. Modeling the threedimensional structure of RNA using discrete nucleotide conformational sets. J Mol Biol 229: 1049-1064.

Gautheret D, Damberger SH, Gutell RR. 1995. Identification of basetriples in RNA using comparative sequence analysis. $J$ Mol Biol 248: $27-43$.

Gutell RR, Power A, Hertz GZ, Putz EJ, Stormo GD. 1992. Identifying constraints on the higher-order structure of RNA: Continued development and application of comparative sequence analysis methods. Nucleic Acids Res 20: 5785-5795.

Holley RW, Apgar J, Everett GA, Madison JT, Marquisee M, Merrill SH, Penswick JR, Zamir A. 1965. Structure of a ribonucleic acid. Science 147: 1462-1465.

Ikawa Y, Shiraishi H, Inoue T. 2000. A small structural element, Pc-J5/5a, plays dual roles in a group IC1 intron RNA. Biochem Biophys Res Commun 274: 259-265.

Jonikas MA, Radmer RJ, Laederach A, Das R, Pearlman S, Herschlag D, Altman RB. 2009. Coarse-grained modeling of large RNA molecules with knowledge-based potentials and structural filters. RNA 15: 189-199.

Kim SH, Quigley GJ, Suddath FL, McPherson A, Sneden D, Kim JJ, Weinzierl J, Rich A. 1973. Three-dimensional structure of yeast phenylalanine transfer RNA: Folding of the polynucleotide chain. Science 179: 285-288.

Kirkpatrick S, Gelatt CD Jr, Vecchi MP. 1983. Optimization by simulated annealing. Science 220: 671-680.

Klingler TM, Brutlag DL. 1993. Detection of correlations in tRNA sequences with structural implications. Proc Int Conf Intell Syst Mol Biol 1: 225-233.

Laederach A, Chan JM, Schwartzman A, Willgohs E, Altman RB. 2007. Coplanar and coaxial orientations of RNA bases and helices. RNA 13: 643-650.

Leontis NB, Stombaugh J, Westhof E. 2002. The non-Watson-Crick base pairs and their associated isostericity matrices. Nucleic Acids Res 30: 3497-3531.

Lescoute A, Westhof E. 2006. Topology of three-way junctions in folded RNAs. RNA 12: 83-93.

Levitt M. 1969. Detailed molecular model for transfer ribonucleic acid. Nature 224: 759-763.

Luebke KJ, Tinoco I Jr. 1996. Sequence effects on RNA bulge-induced helix bending and a conserved five-nucleotide bulge from the group I introns. Biochemistry 35: 11677-11684.

Macke TJ, Case DA. 1998. Modeling unusual nucleic acid structures. ACS Symp Ser Am Chem Socn 1998: 379-393.

Madison JT, Everett GA, Kung HK. 1966. On the nucleotide sequence of yeast tyrosine transfer RNA. Cold Spring Harb Symp Quant Biol 31: 409-416.

Major F, Gautheret D, Cedergren R. 1993. Reproducing the threedimensional structure of a tRNA molecule from structural constraints. Proc Natl Acad Sci 90: 9408-9412.

Martick M, Scott WG. 2006. Tertiary contacts distant from the active site prime a ribozyme for catalysis. Cell 126: 309-320.

Michel F, Jacquier A, Dujon B. 1982. Comparison of fungal mitochondrial introns reveals extensive homologies in RNA secondary structure. Biochimie 64: 867-881.

Murphy FL, Cech TR. 1994. GAAA tetraloop and conserved bulge stabilize tertiary structure of a group I intron domain. J Mol Biol 236: 49-63.

Murphy FL, Wang YH, Griffith JD, Cech TR. 1994. Coaxially stacked RNA helices in the catalytic center of the Tetrahymena ribozyme. Science 265: 1709-1712.

Nissen P, Ippolito JA, Ban N, Moore PB, Steitz TA. 2001. RNA tertiary interactions in the large ribosomal subunit: The A-minor motif. Proc Natl Acad Sci 98: 4899-4903. 
Nkwanta A, Ndifon W. 2009. A contact-waiting-time metric and RNA folding rates. FEBS Lett 583: 2392-2394.

Parisien M, Major F. 2008. The MC-Fold and MC-Sym pipeline infers RNA structure from sequence data. Nature 452: 51-55.

Parisien M, Cruz J, Westhof E, Major F. 2009. New metrics for comparing and assessing discrepancies between RNA 3D structures and models. RNA 15: 1875-1885.

RajBhandary UL, Stuart A, Faulkner RD, Chang SH, Khorana HG. 1966. Nucleotide sequence studies on yeast phenylalanine sRNA. Cold Spring Harb Symp Quant Biol 31: 425-434.

Robertus JD, Ladner JE, Finch JT, Rhodes D, Brown RS, Clark BF, Klug A. 1974. Structure of yeast phenylalanine tRNA at 3 A resolution. Nature 250: 546-551.

Russell R, Herschlag D. 2001. Probing the folding landscape of the Tetrahymena ribozyme: Commitment to form the native conformation is late in the folding pathway. J Mol Biol 308: 839-851.

Smith D, Yarus M. 1989. Transfer RNA structure and coding specificity. II. A D-arm tertiary interaction that restricts coding range. J Mol Biol 206: 503-511.

Snow CD, Nguyen H, Pande VS, Gruebele M. 2002. Absolute comparison of simulated and experimental protein-folding dynamics. Nature 420: 102-106.
Sussman JL, Holbrook SR, Warrant RW, Church GM, Kim SH. 1978. Crystal structure of yeast phenylalanine transfer RNA. I. Crystallographic refinement. J Mol Biol 123: 607-630.

Szewczak AA, Cech TR. 1997. An RNA internal loop acts as a hinge to facilitate ribozyme folding and catalysis. RNA 3: 838-849.

Taketomi H. Ueda Y, Go N. 1975. Studies on protein folding, unfolding, and fluctuations by computer simulation. In J Peptide Protein Res 7: 445-459.

Tyagi R, Mathews DH. 2007. Predicting helical coaxial stacking in RNA multibranch loops. RNA 13: 939-951.

Yang H, Jossinet F, Leontis N, Chen L, Westbrook J, Berman H, Westhof E. 2003. Tools for the automatic identification and classification of RNA base pairs. Nucleic Acids Res 31: 34503460.

Zacharias M, Sklenar H. 1997. Analysis of the stability of looped-out and stacked-in conformations of an adenine bulge in DNA using a continuum model for solvent and ions. Biophys J 73: 29903003.

Znosko BM, Kennedy SD, Wille PC, Krugh TR, Turner DH. 2004. Structural features and thermodynamics of the J4/5 loop from the Candida albicans and Candida dubliniensis group I introns. Biochemistry 43: 15822-15837. 

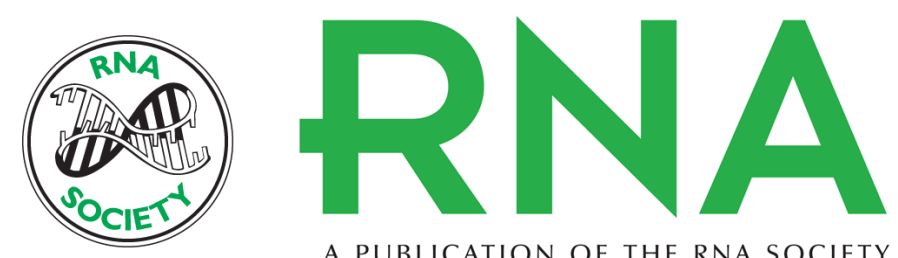

A PUBLICATION OF THE RNA SOCIETY

\section{Turning limited experimental information into 3D models of RNA}

Samuel Coulbourn Flores and Russ B. Altman

RNA 2010 16: 1769-1778 originally published online July 22, 2010

Access the most recent version at doi:10.1261/rna.2112110

Supplemental

Material

References

\section{License}

Email Alerting

Service
http://rnajournal.cshlp.org/content/suppl/2010/07/01/rna.2112110.DC1

This article cites 53 articles, 19 of which can be accessed free at: http://rnajournal.cshlp.org/content/16/9/1769.full.html\#ref-list-1 